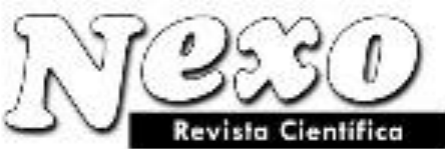

ISSN-E 1995-9516

Universidad Nacional de Ingeniería COPYRIGHT @ (UNI). TODOS LOS DERECHOS RESERVADOS https://revistas.uni.edu.ni/index.php/Nexo https://doi.org/10.5377/nexo.v34i06.13126

\title{
Análisis bibliométrico de las publicaciones científicas nicaragüenses en la Web of Science (2015-2020)
}

\section{Bibliometric analysis of Nicaraguan scientific publications in the Web of Science (2015-2020)}

\author{
Marco Munguía-Mena ${ }^{1}$, Rafael Gamero ${ }^{2}$, Apolinar Picado ${ }^{2 *}$ \\ ${ }^{1}$ Facultad de Electrotecnia y Computación, Universidad Nacional de Ingeniería (UNI) \\ Avenida Universitaria, Managua 11127, Nicaragua \\ ${ }^{2}$ Facultad de Ingeniería Química, Universidad Nacional de Ingeniería (UNI) \\ Avenida Universitaria, Managua 11127, Nicaragua \\ E-mail: picado@kth.se
}

(recibido/received: 31-agosto-2021; aceptado/accepted: 12-diciembre-2021)

\section{RESUMEN}

En este estudio se analizaron las publicaciones científicas nicaragüenses en la Web of Science durante el período 2015-2020. Se recuperaron un total de 780 publicaciones distribuidas en 426 revistas. El análisis de las publicaciones evidenció un ligero crecimiento $(19 \%)$ de la producción científica en el período analizado, una significativa colaboración internacional $(95 \%)$ y la preferencia del inglés $(74 \%)$ como idioma de publicación. El MINSA y la UNAN-Managua fueron las instituciones nicaragüense más productivas, con una contribución combinada del $40 \%$ al total de publicaciones. En cuanto a las áreas de investigación, economía de la empresa es la de mayor contribución, pero el campo temático más productivo corresponde a las ciencias médicas y de la salud.

Palabra claves: Bibliometría; Web of Science; Publicación Científica; Nicaragua

\begin{abstract}
In this study, Nicaraguan scientific publications in the Web of Science during the period 2015-2020 were analysed. A total of 780 publications distributed in 426 journals were retrieved. The analysis of the publications showed a slight growth (19\%) of the scientific production in the analysed period, a significant international collaboration (95\%), and the preference of English (74\%) as the publication language. MINSA and UNAN-Managua were the most productive Nicaraguan institutions, with a combined contribution of $40 \%$ to total publications. Regarding the research areas, business economics is the one with the greatest contribution, but the most productive thematic field corresponds to the medical and health sciences.
\end{abstract}

Keywords: Bibliometric; Web of Science; Scientific Publication; Nicaragua

\footnotetext{
* Autor para la correspondencia
} 


\section{INTRODUCCIÓN}

La ciencia en su devenir histórico ineludiblemente se asocia con sus resultados; entre otros, con sus publicaciones que no son más que una forma de existencia de la propia ciencia. Las publicaciones científicas son depositarias de los conocimientos documentados que la humanidad acumula en cualquier campo del saber; y constituyen la vía fundamental para trasmitir dichos conocimientos ya que la transmisión directa por quienes los poseen a cuantos los necesitan se hace prácticamente imposible (Saavedra-Fernández et al., 2002).

La publicación en una revista de prestigio reconocido asegura la prioridad en la producción de un resultado, acrecienta el crédito académico de un investigador, legitima su actividad y permite la existencia de sistemas de comunicación científica ligados a procesos activos de persuasión, negociación, refutación y modificación, a través de los cuales el significado de las observaciones científicas, al igual que las interpretaciones teóricas, tiende a ser selectivamente construido y reconstruido en el campo científico (Vessuri, 1987).

La misma ciencia urge de mediciones para apreciar sus logros y precisar las fronteras de su estado actual. En este caso, la bibliometría ayuda a precisar, a través de las publicaciones, el desarrollo de una determinada área de la ciencia. Su alcance puede abarcar desde la producción científica de un individuo, grupo o institución de investigación, hasta el comportamiento de todo un sector o campo temático durante un período dado y en un entorno nacional o internacional.

La importancia del análisis bibliométrico es sobradamente conocida pues se concibe como una herramienta especialmente útil a la hora de diseñar estrategias políticas desde el ámbito institucional que redunden en un aumento de visibilidad internacional de las investigaciones científicas y como apoyo para diseñar nuevas líneas de actuación en política científica. El análisis de las publicaciones científicas es el método más extendido y al mismo tiempo más discutido, sobre todo con relación al análisis de la calidad (evaluación cualitativa) más que al de la cantidad (evaluación cuantitativa) (Rojas-Sola y Jordá-Albiñana, 2009).

El reconocimiento de la utilidad del análisis de las publicaciones para el estudio de la actividad de investigación ha llevado a la bibliometría a experimentar un gran desarrollo. En los últimos años los estudios bibliométricos han sido cada vez más requeridos y utilizados no solo para la cuantificación de la producción, sino para otros fines como identificar grupos y áreas de excelencia, asociaciones temáticas, interdisciplinaridad, disciplinas emergentes, redes de colaboración temática, prioridades, etc. Su utilización se ha generalizado en los países más avanzados científicamente. Así lo demuestra su progresiva incorporación a los estudios de evaluación de la actividad investigadora (De Filippo y Fernández, 2002).

En el caso de Nicaragua, Picado et al. (2010) realizaron un estudio bibliométrico utilizando la base de datos Scopus ${ }^{\circledR}$ y analizando el Science Citation Index Expanded para examinar las publicaciones científicas en el período 2007-2009. El estudio determinó que la actividad de investigación alcanzó un crecimiento del 47\% dentro del período analizado; y que el $95 \%$ de las publicaciones se realizaron en colaboración con investigadores provenientes de 62 países. Luego, Monge-Nájera y Ho (2017) estudiaron con más detalle las publicaciones en el Science Citation Index Expanded para el período 1900-2014. Resultados similares fueron obtenidos en términos de colaboración internacional, idioma de publicación, entre otros.

Este trabajo tiene como objetivo analizar, a través de la plataforma Web of Science ${ }^{\mathrm{TM}}$, el comportamiento de las publicaciones científicas nicaragüenses en el período 2015-2020. Este trabajo permitirá conocer el estado actual de las investigaciones en cada área, quienes las realizan, cuáles son las instituciones que apoyan este tipo de investigación y donde son publicadas. 


\section{METODOLOGÍA}

Se utilizó como fuente de información primaria la plataforma Web of Science ${ }^{\mathrm{TM}}$ (Copyright (C) 2021 Clarivate $^{\mathrm{TM}}$ ) que consta de varias bases de datos de búsqueda de literatura diseñadas para respaldar la investigación científica y académica. En este estudio, se utilizó la Web of Science Core Collection que incluye más de 21,000 revistas científicas de alta calidad revisadas por pares y publicadas en todo el mundo (incluidas las revistas de acceso abierto), más de 25,000 actas de conferencias y más de 104,000 libros seleccionados editorialmente.

En la sección de búsqueda de documentos, se realizó una primera búsqueda utilizando el parámetro Nicaragua en el campo de dirección y el parámetro 2015-2020 en el campo de años de publicación. Estos dos parámetros combinados produjeron un total de 1,100 documentos indizados. Luego, se refino la búsqueda utilizando el parámetro Nicaragua en el campo países/regiones, descartando 30 documentos donde el parámetro Nicaragua estaba presente en la dirección (nombre de la calle o vecindario) o en el nombre de la institución, pero que en realidad no eran del país.

Los documentos se revisaron utilizando las herramientas de análisis proporcionadas por la misma plataforma. Además, se decidió no incluir en el presente análisis los siguientes documentos: resúmenes de conferencia (meeting abstracts), editoriales (editorial materials), artículos de revisión (review articles), cartas (letters), criticas de libro (book reviews), poesía (poetry), correcciones (corrections), noticias (news items), capítulos de libro (book chapters), artículos en actas de eventos académicos (proceeding papers) y los artículos en avance (early access), los últimos debido a que estos todavía no tienen todos los detalles bibliográficos disponibles, aunque se pueden citar. Finalmente, quedaron 780 publicaciones científicas para el análisis bibliométrico.

Posteriormente, se elaboró una base de datos con el programa EndNote ${ }^{\mathrm{TM}}$ (Copyright $\left(2020\right.$ Clarivate $^{\mathrm{TM}}$ ). La información de las 780 publicaciones se descargó desde la Web of Science ${ }^{\mathrm{TM}}$ con el formato RIS y se importaron a EndNote ${ }^{\mathrm{TM}}$ con vistas de normalizar los campos considerados en el estudio. Una vez normalizados los campos, se crearon las listas de autores, revistas e instituciones; así como la de los artículos más citados. Estos productos fueron la base de datos empleada para el análisis de las publicaciones científicas nicaragüenses en el período 2015-2020 que se discute en este trabajo. La metodología descrita se aplicó en 3 momentos, entre el 27 de julio y 4 de agosto de 2021, obteniéndose en todos los casos los mismos resultados en cuanto a documentos indizados.

\section{RESULTADOS Y DISCUSIÓN}

El análisis anual de las publicaciones científicas en todas las áreas de ciencia y tecnología se muestra en la Tabla 1. La producción se incrementó en un $18.57 \%$, pasando de 114 artículos en el 2015 a 140 artículos en el 2020. Las 780 publicaciones científicas fueron publicadas en 426 revistas indizadas en la Web of Science $^{\mathrm{TM}}$.

Tabla 1. Número de publicaciones científicas en el período 2015-2020.

\begin{tabular}{ccc}
\hline Año & No. de publicaciones & Porcentaje (\%) \\
\hline 2020 & 140 & 17.95 \\
2019 & 128 & 16.41 \\
2018 & 124 & 15.89 \\
2017 & 144 & 18.46 \\
2016 & 130 & 16.67 \\
2015 & 114 & 14.62 \\
\hline
\end{tabular}


De la Tabla 1 se desprenden diversas observaciones. El número de publicaciones puede considerarse bajo si se toma en cuenta la cantidad de programas de postgrado (i.e. especialidades, maestrías, doctorados) en las diversas áreas de ciencia y tecnología que existen en Nicaragua y el número de estudiantes que egresan por año (CNU, 2020). Esto independientemente de los investigadores adscritos a los centros de investigación, profesores investigadores de las instituciones de educación superior y egresados de licenciatura. La posible causa es que las políticas de fomento a la investigación son casi nulas, no se goza de un gran apoyo presupuestario para el fomento y apoyo a las actividades de investigación, tanto a nivel estatal como a nivel de las instituciones de educación superior. Por ejemplo, el CONACYT prácticamente tiene un accionar nulo en este aspecto. Otra posible causa es la falta de una cultura de investigación ya que no es una tarea prioritaria en las instituciones de educación superior y como resultado, no está desarrollada. Sumado a esto, la gran mayoría (85\%) de los programas de postgrado son programas profesionalizantes y no orientados a la investigación científica.

A nivel básico universitario, a los estudiantes se les enseña poco a investigar. Como resultado, no adquieren experiencia en el quehacer investigativo. Además, el esquema organizativo y legislativo de las instituciones de educación superior continúa privilegiando a la docencia directa, con lo cual se limita el desarrollo de la actividad de investigación.

Se realizó un análisis de las publicaciones según el área de investigación, dividiéndolas para este fin en diferentes áreas de acuerdo con la Web of Science ${ }^{\mathrm{TM}}$. La Tabla 2 muestra las diez (10) áreas con al menos con 30 publicaciones científicas durante el período 2015-2020.

Tabla 2. Distribución de las publicaciones científicas según su área de investigación.

\begin{tabular}{lcc}
\hline \multicolumn{1}{c}{ Área } & No. de publicaciones & Porcentaje (\%) \\
\hline Economía de la empresa & 111 & 14.23 \\
Salud pública, ocupacional y ambiental & 77 & 9.87 \\
Ciencias ambientales y Ecología & 66 & 8.46 \\
Enfermedades infecciosas & 59 & 7.56 \\
Tecnología científica & 56 & 7.18 \\
Ingeniería & 43 & 5.51 \\
Inmunología & 42 & 5.38 \\
Microbiología & 39 & 5.00 \\
Agricultura & 34 & 4.36 \\
Medicina tropical & 30 & 3.85 \\
\hline
\end{tabular}

El análisis confirmó que las ciencias médicas y de la salud y las ciencias naturales fueron los campos temáticos predominantes y con crecimiento en las publicaciones en el período 2015-2020. Además, existió un crecimiento específico en el área de investigación de economía de la empresa debido exclusivamente a la indización en el Emerging Sources Citation Index de la revista electrónica de investigación en ciencias económicas (REICE) de la Universidad Nacional Autónoma de Nicaragua, Managua (UNAN-Managua). En general, la distribución temática de las publicaciones científicas conserva un patrón similar descrito en estudios anteriores (Maltéz et al., 2009; Picado et al., 2010; Monge-Nájera y Ho, 2017) y se ha mantenido sin cambios muy marcados durante la última década.

Con respecto al idioma de publicación, el inglés fue el idioma predominante en las publicaciones con un $74 \%$, seguido del castellano con un $25 \%$, portugués con un $0.5 \%$ y finalmente francés con el $0.5 \%$. Este hecho confirma la elección del inglés como idioma de publicación para la difusión del conocimiento y la visibilidad de los resultados de investigación. En el caso del castellano, existió un aumento en este período 2015-2010 debido mayormente a la indización de algunas revistas latinoamericanas al Emerging Sources Citation Index (e.g. Nexo, REICE, entre otras) que aceptan artículos en inglés, castellano y portugués. 
En el estudio, se identificó que el $61.50 \%$ de las publicaciones científicas fueron publicados en revistas de acceso abierto. Hay que destacar que acceso abierto no implica (no debería implicar) una menor calidad de las publicaciones, ya que la selección de estos se basa también en la revisión por pares. En general, las revistas de acceso abierto favorecen la visibilidad de las publicaciones.

En congruencia con los modelos contemporáneos de investigación colaborativa, las publicaciones contaron con la coautoría de múltiples investigadores, en muchos casos afiliados a instituciones provenientes de diversos países. El 95\% de las publicaciones se realizaron en colaboración con investigadores provenientes de diversos países. En el análisis de las publicaciones en que participaron autores de países extranjeros se destaca el predominio del número de publicaciones realizadas en conjunto con investigadores de instituciones de Estados Unidos, seguido de España, México, entre otros (ver Tabla 3). Es notable la magnitud de la diferencia entre el número de publicaciones en conjunto con investigadores de los Estados Unidos (41.67\% de todas las publicaciones), en comparación con la segunda posición, ocupada por España ( $15.77 \%$ de todas las publicaciones) y las sucesivas. También es notable la amplia heterogeneidad de los países (165 países en total) involucrados, de los cinco continentes, en la coautoría de las publicaciones recopiladas.

Tabla 3. Colaboración internacional en el período 2015-2020.

\begin{tabular}{lcc}
\hline \multicolumn{1}{c}{ País } & No. de publicaciones & Porcentaje $\mathbf{( \% )}$ \\
\hline Estados Unidos & 325 & 41.67 \\
España & 123 & 15.77 \\
México & 118 & 15.13 \\
Costa Rica & 95 & 12.18 \\
Brasil & 83 & 10.64 \\
Inglaterra & 76 & 9.74 \\
Colombia & 71 & 9.10 \\
Suecia & 52 & 6.67 \\
Francia & 50 & 6.41 \\
Guatemala & 50 & 6.41 \\
\hline
\end{tabular}

Las cifras sobre colaboración internacional en las publicaciones que involucran a Nicaragua parecen reflejar, en cierta medida, la proporción global de los aportes al conocimiento por parte de países más avanzados científicamente, pero sugieren también un claro componente de cooperación regional, caso de México, Costa Rica, entre otros. Hay que destacar que Suecia, a pesar de haber terminado el programa de cooperación en materia de investigación con Nicaragua desde finales del 2011, todavía se mantiene como un socio de investigación muy importante, especialmente en el área de las ciencias médicas y de la salud.

Esto refuerza lo analizado por Sancho et al. (2006) que considera de suma importancia la colaboración tanto nacional como internacional para facilitar el desarrollo sostenible, favorecer la movilidad de los investigadores y aumentar el acervo cultural y científico de los participantes. Además, Ríos Gómez y Herrero Solana (2005) confirmaron que los países que más colaboran con América Latina son Estados Unidos, Francia, España, Reino Unido y Alemania, hecho que queda comprobado en este estudio, ya que también los autores de Nicaragua colaboran fundamentalmente con investigadores de los países citados.

En la Tabla 4, se resumió la colaboración internacional con las cinco (5) instituciones extranjeras de más presencia en las publicaciones científicas durante el período 2015-2020. El sistema estatal de la Universidad de California es la institución con más colaboración en el período estudiado, especialmente el campus ubicado en Berkeley. En términos de financiación a la actividad de investigación, la Tabla 5 resume las tres (3) agencias que más han financiado investigaciones reflejadas en las publicaciones científicas en el período 2015-2020. 
Tabla 4. Colaboración de instituciones extranjeras en el período 2015-2020.

\begin{tabular}{lcc}
\hline \multicolumn{1}{c}{ Institución } & No. de publicaciones & Porcentaje (\%) \\
\hline Universidad de California & 186 & 23.85 \\
Universidad de Michigan & 86 & 11.03 \\
Universidad de Carolina del Norte & 83 & 10.64 \\
Universidad de Costa Rica & 27 & 3.46 \\
UNAM-México & 27 & 3.46 \\
\hline
\end{tabular}

Tabla 5. Financiamiento de las investigaciones en el período 2015-2020.

\begin{tabular}{lcc}
\hline \multicolumn{1}{c}{ Agencia } & No. de Publicaciones & Porcentaje (\%) \\
\hline $\begin{array}{l}\text { Institutos Nacionales de Salud de } \\
\text { los Estados Unidos }\end{array}$ & 195 & 14.36 \\
$\begin{array}{l}\text { Departamento de Salud y Servicios } \\
\text { Humanos de los Estados Unidos }\end{array}$ & 124 & 15.89 \\
Comisión Europea & 45 & 5.77 \\
\hline
\end{tabular}

Hay que destacar, que el $98 \%$ de las investigaciones financiadas por estas agencias corresponden a los campos temáticos de las ciencias médicas y de la salud y las ciencias naturales. Esto refuerza el hallazgo que este campo es el de más crecimiento, ya que recibe apoyo financiero y técnico desde agencias externas a Nicaragua a través de redes mundiales/regionales de investigación.

Para el período 2015-2020, se observó que las instituciones nicaragüenses que aporta la mayor proporción de publicaciones científicas son: Ministerio de Salud (MINSA), UNAN-Managua y UNAN-León (ver Tabla 6). La lista de instituciones presentó imprecisiones. Por ejemplo, los diferentes grupos de investigación de una misma institución usaron formas distintas al escribir su adscripción institucional en sus publicaciones. Algunos proporcionaron el nombre completo de la institución, otros mencionaron solo las siglas, usaron abreviaciones incompletas del nombre, mencionaron solo el centro de investigación, la dependencia o facultad e incluso usaron variantes en inglés. Esto se estandarizó manualmente para tener una base de datos curada.

Tabla 6. Instituciones nicaragüenses con más publicaciones científicas en el período 2015-2020.

\begin{tabular}{lcc}
\hline \multicolumn{1}{c}{ Institución } & No. de publicaciones & Porcentaje (\%) \\
\hline MINSA & 154 & 19.74 \\
UNAN-Managua & 153 & 19.62 \\
UNAN-León & 140 & 17.95 \\
Sustainable Sciences Institute (SSI) & 56 & 7.18 \\
UNI & 38 & 4.87 \\
\hline
\end{tabular}

En el campo de las ciencias médicas y de la salud es muy común las redes de coautoría, donde varios profesionales participan en redes mundiales/regionales investigando enfermedades que afectan varios países (e.g. dengue, malaria, lepra, tuberculosis, entre otras). Un ejemplo de estas redes es la red mundial de investigación sobre vacunas en contra del virus chikungunya (Fonseca et al., 2016). Se determinó que el 98\% de las publicaciones científicas asociadas al MINSA provienen de resultados de investigación de alguna red de coautoría. Para facilitar el análisis, se agruparon las distintas instituciones pertenecientes al MINSA que están incluidas como afiliaciones oficiales en las publicaciones científicas. Por ejemplo, el centro nacional de diagnóstico y referencia, centro de salud Sócrates Flores Vivas, hospital escuela Roberto Calderón Gutiérrez, hospital infantil Manuel de Jesús La Mascota, entre otros. 
En el caso de la UNAN-Managua, el 53\% de sus publicaciones científicas provienen de la REICE. Este hallazgo, se puede analizar desde dos aristas. La primera, bastante positiva, es que incrementa la visibilidad de las publicaciones en castellano en la Web of Science ${ }^{\mathrm{TM}}$, ya que probablemente la mayoría de la producción científica nicaragüense es publicada en ese idioma. Este fenómeno fue analizado por Monje-Nájera (2002) para países tropicales, incluyendo Nicaragua. La segunda, algo negativa, es la endogamia editorial y endogamia autoral. Estos dos parámetros de calidad son muy importantes para las revistas publicadas por instituciones de educación superior (Domínguez-Omonte, 2019). La UNAN-León también se benefició de las redes de coautoría internacionales/regionales, ya que un $85 \%$ de las publicaciones provienen de investigaciones de alguna red de coautoría. Es notable la participación de investigadores de Suecia en las publicaciones científicas.

En la Tabla 7 se muestran las publicaciones en el período 2015-2020 con más citas reportadas en la Web of Science $^{\mathrm{TM}}$ (al 27 de julio de 2021). Como se observa, las dos (2) publicaciones con más citas corresponden al campo temático de las ciencias médicas y de la salud, publicados en revistas de corriente principal con factores de impacto altos y los autores nicaragüenses pertenecen a una red de coautoría.

Tabla 7. Publicaciones científicas con más citas en el período 2015-2020.

\begin{tabular}{lcc}
\hline \multicolumn{1}{c}{ Publicación } & Citas & Institución \\
\hline $\begin{array}{l}\text { Shi et al. (2017). Global, regional, and national disease burden } \\
\text { estimates of acute lower respiratory infections due to respiratory }\end{array}$ & 720 & MINSA \\
syncytial virus in young children in 2015: a systematic review and \\
modelling study. The Lancet, 390(10098), 946-958.
\end{tabular}


Para el período 2015-2020, también se realizó la búsqueda de los autores con más publicaciones científicas, así como las instituciones a que pertenecen (ver Tabla 8). El autor con más publicaciones en el período es Ángel Balmaseda, médico de profesión y asociado al MINSA y SSI. El segundo autor con más publicaciones es Guillermina Kuan, médica de profesión y asociada también al MINSA y SSI. Cabe destacar, que ambos autores son parte, por más de 20 años, de un programa de investigación sobre arbovirus (e.g. dengue, zika, chikungunya) dirigido por la profesora Eva Harris ( $\mathrm{PhD}$ en Biología Celular y Molecular) de UC Berkeley. El SSI es una ONG con sede en San Francisco, California con centros en Managua, Nicaragua (2004) y en el Cairo, Egipto (2011); y fundada por la profesora Eva Harris en 1998.

Tabla 8. Autores con más publicaciones científicas en el período 2015-2020.

\begin{tabular}{lcc}
\hline \multicolumn{1}{c}{ Autor } & No. de publicaciones & Institución \\
\hline Ángel Balmaseda & 89 & MINSA, SSI \\
Guillermina Kuan & 39 & MINSA, SSI \\
Lionel Gresh & 38 & SSI \\
Sergio Ojeda & 21 & SSI \\
Nery Sánchez & 21 & SSI \\
Filemón Bucardo & 16 & UNAN-León \\
Raquel Burger-Calderón & 10 & SSI \\
Roger López & 10 & MINSA, SSI \\
\hline
\end{tabular}

Tal como lo han apuntado varios estudios, la ciencia en Latinoamérica ha experimentado un incremento en los últimos años en términos de publicaciones científicas en bases de datos internacionales (e.g. WoS, Scopus), no obstante, para la evaluación exhaustiva de las publicaciones científicas de Nicaragua, y de muchos países latinoamericanos, es necesario también emplear bases de datos regionales (e.g. Scielo) y nacionales, con el objetivo de capturar la mayor cantidad de publicaciones generadas por las instituciones nicaragüenses. Sin embargo, el uso de la plataforma Web of Science ${ }^{\mathrm{TM}}$ es lo más aceptado para el análisis bibliométrico para identificar y monitorear las tendencias de investigación (Clarivate, 2021).

\section{CONCLUSIONES}

El crecimiento en el número total de publicaciones generadas por año, aunque sostenido, es bastante leve y no parece reflejar un desarrollo acelerado, como el que han mostrado algunos países latinoamericanos que implementaron un fuerte apoyo político-económico para la ciencia (e.g. México, Chile). El crecimiento observado podría más bien constituir un indicador de desarrollo pasivo de la actividad científica. Lo anterior parecería concordar con el hecho de que las políticas de apoyo estatal a las actividades de investigación han sido pocas en las últimas dos décadas. El establecimiento de políticas estatales claras, eficientes y sostenidas en lo referente al apoyo de la investigación científica, será fundamental para determinar la dirección futura de este aparentemente frágil componente de la sociedad nicaragüense, insuficiente pero indispensable para nutrir la cultura, la tecnología y el desarrollo de toda la nación.

\section{CONFLICTO DE INTERÉS}

Los autores declaran que no existe conflicto de interés con respecto a la publicación de este artículo.

\section{AGRADECIMIENTOS}

Este trabajo no recibió ninguna subvención específica de agencias de financiamiento en los sectores público, comercial o sin fines de lucro. A. Picado agradece al Real Instituto de Tecnología (KTH), Suecia. 


\section{REFERENCIAS}

Clarivate (2021, August 30). Web of Science platform: Web of Science: Summary of Coverage. URL: https://clarivate.libguides.com/webofscienceplatform/coverage

Consejo Nacional de Universidades (CNU) (2020). Información estadística de las universidades miembros del CNU año 2019. Informe Técnico. Managua, Nicaragua.

De Filippo, D., \& Fernández, M.T. (2002). Bibliometría: importancia de los indicadores bibliométricos. En M. Albornoz (Ed.), El estado de la ciencia 2002 (pp. 1-10). RICYT, Argentina.

Domínguez-Omonte, C.-B. (2019). El reto de la gestión editorial de revistas científicas, la "endogamia" editorial, y autoral. Revista Científica Ciencia Médica, 22(1), 3-4.

Fonseca, B.d., Sampaio, R.B., Fonseca, M.V.d., \& Zicker, F. (2016). Co-authorship network analysis in health research: method and potential use. Health Research Policy and Systems, 14(1), 34. doi: $\underline{10.1186 / \mathrm{s} 12961-016-0104-5}$

Maltéz, J.M., Rojas, S., Picado, A., \& Munguía-Mena, M. (2009). Estudio del impacto de las investigaciones del área de las ciencias médicas y de la salud de las universidades miembros del Consejo Nacional de Universidades (CNU). Informe Técnico. doi: 10.13140/RG.2.2.27883.28963

Monge-Nájera, J. (2002). Cómo hacer ciencia en los trópicos. Revista Biología Tropical, 50(3-4).

Monge-Nájera, J., \& Ho, Y.-S. (2017). Bibliometrics of Nicaraguan publications in the Science Citation Index Expanded. Revista Biología Tropical, 65(2), 643-655. doi: 10.15517/rbt.v65i2.23985

Picado, A., Munguía-Mena, M., \& Rojas, S. (2010). Análisis bibliométrico de las publicaciones científicas de Nicaragua en el Science Citation Index Expanded: trienio 2007-2009. In Proceedings of the 1st LatinAmerican Multidisciplinary Congress of Applied Sciences (COMCAPLA 2010). Paper No. AMD101. doi: $\underline{10.13140 / R G .2 .1 .1669 .2887}$

Ríos Gómez, C., \& Herrero Solana, V. (2005). La producción científica latinoamericana y la ciencia mundial: una revisión bibliográfica (1989-2003). Revista Interamericana de Bibliotecología, 28(1), 43-61.

Saavedra-Fernández, Ó., Sotolongo-Aguilar, G., \& Guzmán-Sánchez, M.V. (2002). Medición de la producción científica en América Latina y el Caribe en el campo agrícola y afines: un estudio bibliométrico. Revista Española De Documentación Científica, 25(2), 151-161. doi: 10.3989/redc.2002.v25.i2.96

Sancho, R., Morillo, F., De Filippo, D., Gómez, I., \& Fernández, M.T. (2006). Indicadores de colaboración científica inter-centros en los países de América Latina. Interciencia, 31(4), 284-292.

Rojas-Sola, J.I., \& Jordá-Albiñana, B. (2009). Análisis bibliométrico de las publicaciones científicas españolas en la categoría materials science, ceramics de la base de datos JCR (SCI) (1997-2008). Boletín de la Sociedad Española de Cerámica y Vidrio, 48(5), 255-260.

Vessuri, H.M.V. (1987). La revista científica periférica. El caso de Acta Científica Venezolana. Interciencia, 12(3), 124-134. 